\title{
Do Spectroscopic Dense Gas Fractions Track Molecular Cloud Surface Densities?
}

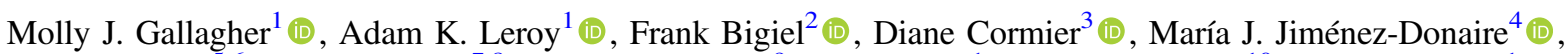

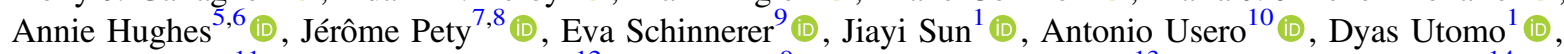 \\ Alberto Bolatto $^{11}$ (10), Mélanie Chevance ${ }^{12}$, Chris Faesi ${ }^{9}$ (i), Simon C. O. Glover ${ }^{13}$ (1) , Amanda A. Kepley ${ }^{14}$ (i),

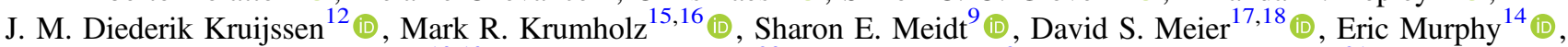 \\ Miguel Querejeta ${ }^{10,19}$ (1) , Erik Rosolowsky ${ }^{20}$ (1) , Toshiki Saito $^{9}$ (D), and Andreas Schruba ${ }^{21}$ \\ ${ }^{1}$ Department of Astronomy, The Ohio State University 4055 McPherson Laboratory, 140 West 18th Avenue, Columbus, OH 43210, USA; gallagher.674@osu.edu \\ 2 AIfA, Universität Bonn, Auf dem Hügel 71, D-53121 Bonn, Germany \\ ${ }^{3}$ AIM, CEA, CNRS, Université Paris-Saclay, Université Paris Diderot, Sorbonne Paris Cité, F-91191 Gif-sur-Yvette, France \\ ${ }^{4}$ Harvard-Smithsonian Center for Astrophysics, 60 Garden Street, Cambridge, MA 02138, USA \\ ${ }^{5}$ CNRS, IRAP, 9 av. du Colonel Roche, BP 44346, F-31028 Toulouse cedex 4, France \\ ${ }^{6}$ Université de Toulouse, UPS-OMP, IRAP, F-31028 Toulouse cedex 4, France \\ ${ }^{7}$ Institut de Radioastronomie Millimètrique (IRAM), 300 Rue de la Piscine, F-38406 Saint Martin d'Hères, France \\ ${ }^{8}$ Observatoire de Paris, 61 Avenue de l'Observatoire, F-75014 Paris, France \\ ${ }_{10}^{9}$ Max Planck Institute für Astronomie, Königstuhl 17, D-69117, Heidelberg, Germany \\ ${ }^{10}$ Observatorio Astronómico Nacional (IGN), C/Alfonso XII, 3, E-28014 Madrid, Spain \\ ${ }^{11}$ Department of Astronomy, University of Maryland, College Park, MD 20742-2421, USA \\ ${ }_{12}^{12}$ Astronomisches Rechen-Institut, Zentrum für Astronomie der Universität Heidelberg, Mönchhofstraße 12-14, D-69120 Heidelberg, Germany \\ ${ }^{13}$ Institute für theoretische Astrophysik, Zentrum für Astronomie der Universität Heidelberg, Albert-Ueberle Str. 2, D-69120 Heidelberg, Germany \\ ${ }^{14}$ National Radio Astronomy Observatory, 520 Edgemont Road, Charlottesville, VA 22903-2475, USA \\ ${ }^{15}$ Research School of Astronomy \& Astrophysics, Australian National University, Canberra, ACT 2611, Australia \\ ${ }^{16}$ Centre of Excellence for Astronomy in Three Dimensions (ASTRO-3D), Australia \\ ${ }^{17}$ Department of Physics, New Mexico Institute of Mining and Technology, 801 Leroy Place, Soccoro, NM 87801, USA \\ ${ }_{18}$ National Radio Astronomy Observatory, P.O. Box O, 1003 Lopezville Road, Socorro, NM, 87801, USA \\ ${ }^{19}$ European Southern Observatory, Karl-Schwarzschild-Straße 2, D-85748 Garching bei München, Germany \\ ${ }^{20}$ Department of Physics, University of Alberta, Edmonton, AB T6G 2E1, Canada \\ ${ }^{21}$ Max-Planck-Institut für extraterrestrische Physik, Giessenbachstraße 1, D-85748 Garching, Germany \\ Received 2018 August 22; revised 2018 October 26; accepted 2018 November 14; published 2018 November 30
}

\begin{abstract}
We use Atacama Large Millimeter/submillimeter Array and Institute for Radio Astronomy in the Millimeter $30 \mathrm{~m}$ telescope data to investigate the relationship between the spectroscopically traced dense gas fraction and the cloudscale $(120 \mathrm{pc})$ molecular gas surface density in five nearby, star-forming galaxies. We estimate the dense gas mass fraction at 650 and $2800 \mathrm{pc}$ scales using the ratio of $\mathrm{HCN}(1-0)$ to $\mathrm{CO}(1-0)$ emission. We then use highresolution $(120 \mathrm{pc}) \mathrm{CO}(2-1)$ maps to calculate the mass-weighted average molecular gas surface density within 650 or $2770 \mathrm{pc}$ beam where the dense gas fraction is estimated. On average, the dense gas fraction correlates with the mass-weighted average molecular gas surface density. Thus, parts of a galaxy with higher mean cloud-scale gas surface density also appear to have a larger fraction of dense gas. The normalization and slope of the correlation do vary from galaxy to galaxy and with the size of the regions studied. This correlation is consistent with a scenario where the large-scale environment sets the gas volume density distribution, and this distribution manifests in both the cloud-scale surface density and the dense gas mass fraction.
\end{abstract}

Key words: galaxies: ISM - galaxies: star formation - ISM: clouds - ISM: molecules - ISM: structure

\section{Introduction}

Star formation is tied to the presence of dense gas. We observe this in the Milky Way, where stars form primarily in dense substructures within molecular clouds (e.g., Lada \& Lada 2003; Heiderman et al. 2010; Lada et al. 2010, 2012; André et al. 2014; Evans et al. 2014). We also observe this in external galaxies. There the dense gas mass, traced by high effective critical density molecular lines, correlates with the star formation rate (e.g., see Gao \& Solomon 2004; García-Burillo et al. 2012; Chen et al. 2015; Usero et al. 2015; Bigiel et al. 2016; Gallagher et al. 2018; Kepley et al. 2018). Both Galactic and extragalactic observations also indicate that gas volume density and its relationship to star formation change as a function of environment (e.g., Gao \& Solomon 2004; Longmore et al. 2013; Kruijssen et al. 2014; Usero et al. 2015; Bigiel et al. 2016; Gallagher et al. 2018). Measuring the gas volume density across many environments is key to understanding what drives these variations.
Observers use two main methods to gauge the distribution of volume densities in the molecular interstellar medium (ISM) of other galaxies. First, one can estimate the surface density of molecular clouds by imaging a line that traces the bulk molecular gas mass, such as $\mathrm{CO}$ emission (e.g., Hughes et al. 2013; Colombo et al. 2014). Given an estimate of the line-ofsight depth, e.g., from a cloud size or adopted scale height, we can convert this surface density to a volume density. This method requires high physical resolution $(\lesssim 100 \mathrm{pc})$ to avoid bias from beam dilution.

One can also infer the distribution of gas volume density ${ }^{22}$ from observations of multiple molecular emission lines that are excited at different effective critical densities, $n_{\text {eff }}$ (e.g., Gao \& Solomon 2004; García-Burillo et al. 2012). To first order, the luminosity of a line traces the mass of gas above its $n_{\text {eff. }}$.

\footnotetext{
22 Unless otherwise stated, "density" refers to gas volume density throughout this Letter.
} 
Changes in the ratio of intensities between lines with different $n_{\text {eff }}$ can indicate a changing ratio in the mass above each density (though there are subtleties; see Krumholz \& Thompson 2007; Leroy et al. 2017). This method constrains the volume density distribution within the beam without the need to resolve individual clouds. In the simple case of a bulk gas tracer (here we use $\mathrm{CO}(1-0)$ with $n_{\mathrm{eff}} \approx 1 \times 10^{2} \mathrm{~cm}^{-3}$ ) and a dense gas tracer (here we use $\operatorname{HCN}(1-0)$ with $n_{\mathrm{eff}} \approx 5 \times 10^{3} \mathrm{~cm}^{-3}$, e.g., Onus et al. 2018), this method traces the dense gas fraction $\left(f_{\text {dense }}\right)$. Because high effective critical density lines also tend to be faint, this method has been mostly employed in low-resolution, high-sensitivity data sets, which average over scales much larger than that of an individual cloud (e.g., Gao \& Solomon 2004; Chen et al. 2015; Usero et al. 2015; Bigiel et al. 2016; Gallagher et al. 2018).

These two methods trace density in different ways at different scales but they should be related. If the mean volume density (and thus also the mean surface density) of a cloud increases or decreases, then we might expect the fraction of gas above some effective density (e.g., that of $\mathrm{HCN}$ ) to rise or drop in parallel. Exactly this prediction arises from turbulent cloud models (e.g., Padoan \& Nordlund 2002; Krumholz \& Thompson 2007; Federrath \& Klessen 2013). In these models, increasing the mean volume density of a cloud "slides" the density distribution to a higher range of values and thus should increase $f_{\text {dense. }}$. This Letter represents the first observational test of this correlation.

In this Letter, we leverage the results of recent observing campaigns using the Institute for Radio Astronomy in the Millimeter range (IRAM) $30 \mathrm{~m}^{23}$ and the Atacama Large Millimeter/submillimeter Array (ALMA) to compare these two density estimates. We estimate the cloud-scale molecular gas surface density from the Physics at High Angular resolution in Nearby GalaxieS (PHANGS)-ALMA survey ${ }^{24}$ (PIs: E. Schinnerer \& A. K. Leroy et al. 2018, in preparation). PHANGS-ALMA is mapping $\mathrm{CO}(2-1)$ at high resolution $(\sim 100 \mathrm{pc})$ across 74 nearby galaxies. We compare this to $f_{\text {dense }}$ estimated using $\mathrm{HCN}(1-0)$ and $\mathrm{CO}(1-0)$ (hereafter referred to as $\mathrm{HCN}$ and $\mathrm{CO}$, respectively) maps from ALMA (Gallagher et al. 2018) and the IRAM Large Program EMPIRE (Bigiel et al. 2016, M. Jimenez Donaire et al. 2018, in preparation).

Section 2 summarizes how we calculate the $\mathrm{HCN} / \mathrm{CO}$ ratio (Section 2.1), the characteristic cloud-scale CO intensity with a larger $\sim$ kpc-scale aperture (Section 2.2), and the mean relation between the two (Section 2.3). Section 3 presents the observed correlation (Section 3.1) and discusses its physical implications (Section 3.2). We compare our results to simple density distribution models (Section 3.3). Section 4 summarizes our findings.

\section{Data and Methods}

Table 1 lists our targets. We consider two samples, based on the availability of $\mathrm{HCN}(1-0)$ data, which we analyze separately. The EMPIRE sample has $34^{\prime \prime}$ resolution HCN maps from the IRAM $30 \mathrm{~m}$. The ALMA sample has $8^{\prime \prime} \mathrm{HCN}$ maps. NGC 3627, NGC 4254, and NGC 4321 appear in both

\footnotetext{
${ }^{23}$ This work is partially based on observations carried out with the IRAM $30 \mathrm{~m}$ telescope. IRAM is supported by INSU/CNRS (France), MPG (Germany) and IGN (Spain)

${ }^{24}$ http://phangs.org
}

samples. NGC 3351 appears only in the ALMA sample and NGC 5194 appears only in the EMPIRE sample.

\section{1. $\mathrm{HCN}(1-0)$ to $\mathrm{CO}(1-0)$ Ratio}

EMPIRE HCN Data: EMPIRE (Bigiel et al. 2016) used the IRAM $30 \mathrm{~m}$ telescope to map HCN emission from nine galaxies, four of which have high-resolution CO maps suitable for our experiment. The EMPIRE maps cover the whole starforming disk of each galaxy, but have relatively poor (34") angular resolution.

M. Jimenez Donaire et al. (2018, in preparation) present a full description of EMPIRE, including the new CO (1-0) maps (see also Cormier et al. 2018), which we pair with the HCN maps to measure the $\mathrm{HCN}(1-0)$ to $\mathrm{CO}(1-0)$ ratio. Briefly, EMPIRE uses the IRAM $30 \mathrm{~m}$ in on-the-fly mapping mode to cover the area of active star formation in each target (see Figure 1). Observations were conducted from 2012 to 2016. The data were calibrated using GILDAS, extracted at $4 \mathrm{~km} \mathrm{~s}^{-1}$ spectral resolution and then further reduced using an in-house pipeline. The pipeline fits and subtracts a second-order polynomial baseline, avoiding regions of the spectrum known to have bright $\mathrm{CO}$ emission. It rejects spectra with measured noise that is significantly larger than that predicted by the radiometer equation. Then it projects the data on to grids with pixel size of $4^{\prime \prime}$. The adopted gridding kernel convolved with the IRAM $30 \mathrm{~m}$ beam yields a final angular resolution of 34". Finally, the pipeline converts the data to main beam temperature units, assuming main beam and forward efficiencies of 0.78 and 0.94 (Carter et al. 2012, IRAM calibration papers).

ALMA HCN Data: Gallagher et al. (2018) mapped HCN emission from four galaxies and assembled matched-resolution CO (1-0) maps from the literature ${ }^{25}$ The ALMA maps cover out to $r_{\text {gal }}=3.5-6 \mathrm{kpc}$, a smaller area than EMPIRE (Figure 1), but they have a sharper $8^{\prime \prime}$ resolution.

We take $\mathrm{CO}$ observations from the Berkeley-Illinois-Maryland Association Survey of Nearby Galaxies (BIMA SONG; Helfer et al. 2003) for NGC 3351 and NGC 3627. These cubes include data from both the BIMA interferometer and short spacing data from the NRAO $12 \mathrm{~m}$ single-dish telescope on Kitt Peak. We take interferometric $\mathrm{CO}$ observations from the Combined Array for Research in Millimeter-wave Astronomy Survey Toward IR-bright Nearby Galaxies (CARMA STING; Rahman et al. 2015) for NGC 4254. We combine this with single-dish data from the CO extension to the IRAM EMPIRE survey (Cormier et al. 2018, M. Jiménez-Donaire et al. 2018, in preparation). We take CO data from the ALMA science verification program for NGC 4321. This includes both main $12 \mathrm{~m}$ array and Atacama Compact Array (ACA) short spacing and total power data. We take the CO data from EMPIRE for NGC 5194.

ALMA observed HCN (1-0) using a seven-field mosaic centered on the nucleus of the galaxy. The data were reduced using the CASA (McMullin et al. 2007) package and observatory-provided calibration scripts. They were then imaged using natural weighting with a small $u-v$ taper and a velocity resolution of $10 \mathrm{~km} \mathrm{~s}^{-1}$. Using the CASA task feather, the ALMA cubes were combined with the IRAM $30 \mathrm{~m}$ maps (mostly from EMPIRE) to correct for missing short

\footnotetext{
25 The matched-resolution CO (1-0) maps come from ALMA, CARMA, and BIMA. All include short and zero spacing data. Gallagher et al. (2018) give more details.
} 
Table 1

Galaxy Sample

\begin{tabular}{|c|c|c|c|c|c|}
\hline Galaxy & HCN Survey & High-resolution $\mathrm{CO}$ & $\begin{array}{l}\text { Distance } \\
(\mathrm{Mpc})\end{array}$ & $\begin{array}{c}8^{\prime \prime} \text { Resolution } \\
(\mathrm{kpc})\end{array}$ & $\begin{array}{c}34^{\prime \prime} \text { Resolution } \\
(\mathrm{kpc})\end{array}$ \\
\hline NGC 3351 & ALMA & PHANGS-ALMA & 10.0 & 0.39 & $\ldots$ \\
\hline NGC 3627 & ALMA, EMPIRE & PHANGS-ALMA & 8.28 & 0.32 & 1.36 \\
\hline NGC 4321 & ALMA, EMPIRE & PHANGS-ALMA & 15.2 & 0.59 & 2.51 \\
\hline NGC 5194 & EMPIRE & PAWS & 8.39 & $\ldots$ & 1.38 \\
\hline
\end{tabular}

Note. HCN Data: ALMA—Gallagher et al. (2018), EMPIRE_-IRAM 30 m Large Program (Bigiel et al. 2016, M. Jimenez Donaire et al. 2018, in preparation). Highresolution CO Data: PdBI Arcsecond Whirlpool Survey (PAWS) CO (1-0)—Schinnerer et al. (2013). PHANGS-ALMA CO (2-1)—A. K. Leroy et al. (2018, in preparation) and Sun et al. (2018). Distance: adopted distance in Mpc from the Extragalactic Distance Database (Tully et al. 2009). $8^{\prime \prime}\left(34^{\prime \prime}\right)$ Resolution: physical resolution corresponding to $8^{\prime \prime}$ and $34^{\prime \prime}$ at our adopted distances.

spacing data. The resulting cubes were convolved to a resolution of $8^{\prime \prime}$, chosen to match archival $\mathrm{CO}$ and infrared data (see Gallagher et al. 2018). The statistical noise in each $10 \mathrm{~km} \mathrm{~s}^{-1}$ channel is $\sim 5-10 \mathrm{mK}$.

For each sample, we convolve the HCN $(1-0)$ and CO $(1-0)$ data from the literature for all targets to a common physical resolution set by the physical beam size at the most distant target. This is $650 \mathrm{pc}$ for ALMA and $2770 \mathrm{pc}$ for EMPIRE. At this common resolution, we measure $\mathrm{HCN} / \mathrm{CO}$, the ratio of the $\mathrm{HCN}(1-0)$ to $\mathrm{CO}(1-0)$ integrated intensities. We constructed an integrated intensity map of $\mathrm{HCN}$ using a mask defined in position-position-velocity space from the $\mathrm{CO}(1-0)$ cube. We then measure $\mathrm{HCN} / \mathrm{CO}$ everywhere within the fields of view that CO is detected (see Gallagher et al. 2018).

\subsection{Average Cloud-scale CO Intensity in each HCN Beam}

We estimate the cloud-scale surface density from PHANGSALMA CO (2-1) and (for NGC 5194) PAWS CO (1-0) data at $120 \mathrm{pc}$ resolution (see Figure 1). PHANGS-ALMA produces CO (2-1) line maps with $\sim 1^{\prime \prime}-1$ ".5 resolution, $2.5 \mathrm{~km} \mathrm{~s}^{-1}$ velocity resolution, $\sim 0.1 \mathrm{~K}$ noise per channel, and including short spacing and total power information from ALMA's Morita ACA. Data reduction and imaging for PHANGSALMA are described in A. K. Leroy et al. (2018, in preparation). Details regarding the creation of moment maps, noise, and completeness for the four targets studied here appear in Sun et al. (2018). Given the distances and angular resolutions of these data, $120 \mathrm{pc}$ represents the common physical resolution for our high-resolution $\mathrm{CO}$ data. We convolve all four $\mathrm{CO}(2-1)$ maps to share this common physical resolution.

For NGC 5194, we also convolve the PAWS CO (1-0) moment-zero map (Pety et al. 2013; Schinnerer et al. 2013) to $120 \mathrm{pc}$ resolution. To place these measurements on the same CO (2-1) intensity scale as our other targets, we then multiply the PAWS CO (1-0) intensities by a typical CO (2-1)/CO (1-0) ratio of 0.7 (uncertain by \pm 0.15 dex, see Leroy et al. 2013).

We measure $\mathrm{HCN} / \mathrm{CO}$ at coarser resolution than we measure the $\mathrm{CO}(2-1)$ intensity, $I_{\mathrm{CO}(2-1)}$. To connect the two measurements, we calculate the intensity-weighted average $I_{\mathrm{CO}(2-1)}$ within each larger $\mathrm{HCN}$ beam. This weighted average, $\left\langle I_{\mathrm{CO}(2-1)}\right\rangle$, measures the mean 120 pc resolution $I_{\mathrm{CO}(2-1)}$ from which CO photons emerge. Formally,

$$
\left\langle I_{\mathrm{CO}(2-1)}\right\rangle=\frac{\left(I_{\mathrm{CO}(2-1)}\right)^{2} * \Omega}{I_{\mathrm{CO}(2-1)} * \Omega} .
$$

Here, $I_{\mathrm{CO}(2-1)}$ is the $\mathrm{CO}$ map at $120 \mathrm{pc}$ resolution, the asterisk represents convolution, and $\Omega$ indicates the Gaussian kernel used to change the resolution of the map from $120 \mathrm{pc}$ to the final resolution $(650 \mathrm{pc}$ for the ALMA sample and $2770 \mathrm{pc}$ for the EMPIRE sample).

$\left\langle I_{\mathrm{CO}(2-1)}\right\rangle$ is the expectation value of $\mathrm{CO}$ intensity weighted by itself within each coarser $\mathrm{HCN}$ beam. In practice, given some conversion between light and mass (i.e., $\left.\alpha_{\mathrm{CO}}\right),\left\langle I_{\mathrm{CO}(2-1)}\right\rangle$ captures the mass-weighted $120 \mathrm{pc}$ resolution surface density of molecular gas inside each larger HCN beam, $\left\langle\Sigma_{\text {mol }}\right\rangle$.

The advantage of this approach, which is discussed at length by Leroy et al. (2016; and see Ossenkopf \& Mac Low 2002; Utomo et al. 2018) is that it preserves the high-resolution information and down-weights empty regions. Compared to the unweighted average, this intensity-weighted average, $\left\langle I_{\mathrm{CO}(2-1)}\right\rangle$ is 0.6 dex higher at $650 \mathrm{pc}$ resolution and 1.4 dex higher at $2770 \mathrm{pc}$ resolution. The intensity weighting is not equivalent to smoothing, as it effectively leverages the high-resolution information and yields characteristic surface densities that are 2-30 times higher than smoothed maps. The difference reflects beam dilution due to the large amount of empty space in the CO maps, which is also visible from Figure 1.

Uncertainty: We estimate the uncertainty in $\left\langle I_{\mathrm{CO}(2-1)}\right\rangle$ via a Monte Carlo calculation. We begin with the original CO $(2-1)$ cubes, add randomly generated Gaussian noise with the correct mean amplitude, and then run these noise-added cubes through our full analysis procedure. We repeat this process 100 times, and calculate the standard deviation in $\left\langle I_{\mathrm{CO}(2-1)}\right\rangle$ over all realizations. The mean error calculated in this way is $\sim 2 \mathrm{~K} \mathrm{~km} \mathrm{~s}^{-1}$. As a result, all $\left\langle I_{\mathrm{CO}(2-1)}\right\rangle$ values in this Letter have a signal-to-noise ratio $(\mathrm{S} / \mathrm{N})>5$.

The distances to our targets are uncertain by $\sim 10 \%-30 \%$. The angular scale corresponding to $120 \mathrm{pc}$ is correspondingly uncertain, adding an additional uncertainty to our calculation. Using the same data that we use here, Sun et al. (2018) showed that changing from a physical resolution of $80-120$ pc (i.e., by $>30 \%$ ) alters the mean $I_{\mathrm{CO}(2-1)}$ in a galaxy by $\sim 0.05-0.1$ dex.

\subsection{Binned Relation}

Our EMPIRE and ALMA surveys only detect HCN along individual lines of sight at high signal-to-noise in the brightest regions of our targets. However, our data also contain a large amount of information at lower signal-to-noise.

We recover $\left\langle I_{\mathrm{CO}(2-1)}\right\rangle$ at high signal-to-noise across a wide area. Therefore, to access the fainter $\mathrm{HCN}$ emission, we measure 

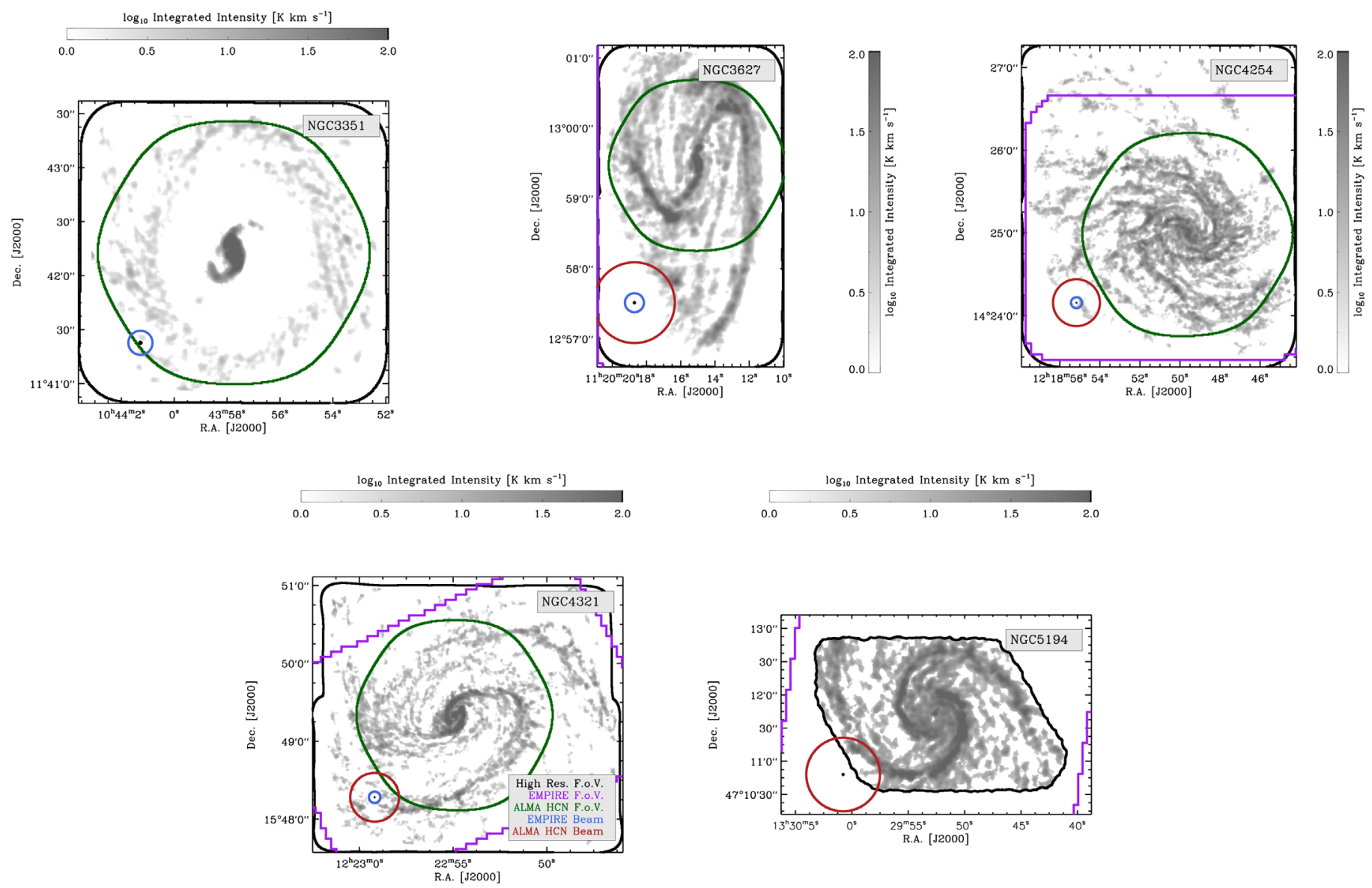

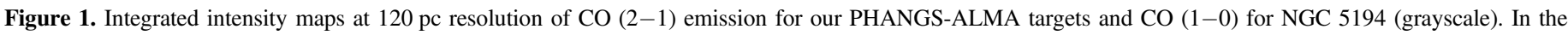

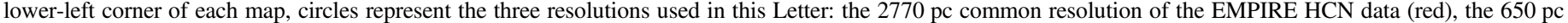

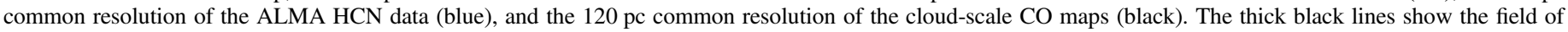
view of the high-resolution map, and the green and purple lines represent the fields of view of the ALMA (green) and EMPIRE (purple) HCN data.

the average $\mathrm{HCN} / \mathrm{CO}$ in bins of $\left\langle I_{\mathrm{CO}(2-1)}\right\rangle$. We report the integrated $\mathrm{HCN}$ divided by the integrated $\mathrm{CO}$ in each bin, with the statistical uncertainty on this binned ratio propagated from the original maps following Gallagher et al. (2018). This binning increases the signal-to-noise in $\mathrm{HCN} / \mathrm{CO}$ via averaging and extends the dynamic range in our measured correlation dramatically.

Following Gallagher et al. (2018), our HCN integrated intensity maps are created by integrating the cube over the region with bright $\mathrm{CO}$ emission, whether or not that region shows HCN emission at high signal-to-noise. As a result, this averaging approach is almost equivalent to spectral stacking using the CO velocity field as a prior (as in Schruba et al. 2011; Jiménez-Donaire et al. 2017). We verify this by comparing the two approaches directly. After shifting the HCN cubes to the local mean $\mathrm{CO}$ velocity and averaging, we derive stacked line ratios in bins of $\left\langle I_{\mathrm{CO}}\right\rangle$. On average, the spectral stacking yields the same results as our mask-and-average approach within $\sim 10 \%$, with no systematic offset.

\subsection{Interpretation in Terms of Physical Quantities}

We report the observed $\mathrm{HCN} / \mathrm{CO}$ ratio as a function of $\left\langle I_{\mathrm{CO}(2-1)}\right\rangle$. These quantities are interesting because they trace the fraction of dense gas and the mean surface density of molecular clouds. Adopting simple translations from observables, we indicate these two physical quantities on the alternative right and top axes of Figures 2 and 3.

To translate $\mathrm{HCN} / \mathrm{CO}$ to $f_{\text {dense }}$, we assume $\alpha_{\mathrm{CO}(1-0)}=$ $4.35 M_{\odot} \mathrm{pc}^{-2}\left(\mathrm{~K} \mathrm{~km} \mathrm{~s}^{-1}\right)^{-1}$ (Bolatto et al. 2013) and a more uncertain $\alpha_{\mathrm{HCN}} \approx 14 M_{\odot} \mathrm{pc}^{-2}\left(\mathrm{~K} \mathrm{~km} \mathrm{~s}^{-1}\right)^{-1}$ to convert $\mathrm{HCN}$ to the mass of gas above a density of $n_{\mathrm{H} 2} \approx 5 \times 10^{3} \mathrm{~cm}^{-3}$ (Onus et al. 2018). For comparison, many previous studies have assumed $\alpha_{\mathrm{HCN}}=10 M_{\odot} \mathrm{pc}^{-2}\left(\mathrm{~K} \mathrm{~km} \mathrm{~s}^{-1}\right)^{-1}$ for gas above $3 \times 10^{4} \mathrm{~cm}^{-3}$ (following Gao \& Solomon 2004).

Both the density of gas traced by $\mathrm{HCN}(1-0)$ and the conversion from $\mathrm{HCN}$ emission to a dense gas mass remain uncertain. The effective critical density of $\mathrm{HCN}$ changes as a function of temperature and optical depth, which are hard to measure (Jiménez-Donaire et al. 2017). Moreover, gas at densities below the effective critical density still emits HCN, rendering the density traced by $\mathrm{HCN}$ a product of the emissivity and density distribution (Leroy et al. 2017). For more discussion, see Gao \& Solomon (2004), Usero et al. (2015), Leroy et al. (2017), Onus et al. (2018), and Gallagher et al. (2018), as well as the Milky Way studies by Kauffmann et al. (2017), Mills \& Battersby (2017), and Pety et al. (2017).

Similarly, we report $\left\langle I_{\mathrm{CO}(2-1)}\right\rangle$ as our primary measurement and the $120 \mathrm{pc}$ resolution molecular gas surface density, $\left\langle\Sigma_{\mathrm{mol}}\right\rangle$, as an alternative axis. For a typical $\mathrm{CO}(2-1) / \mathrm{CO}(1-0)$ ratio of 0.7 , the Galactic CO-to- $\mathrm{H}_{2}$ conversion factor is $\alpha_{\mathrm{CO}(2-1)} \approx 6.2 M_{\odot} \mathrm{pc}^{-2}\left(\mathrm{~K} \mathrm{~km} \mathrm{~s}^{-1}\right)^{-1}$. 


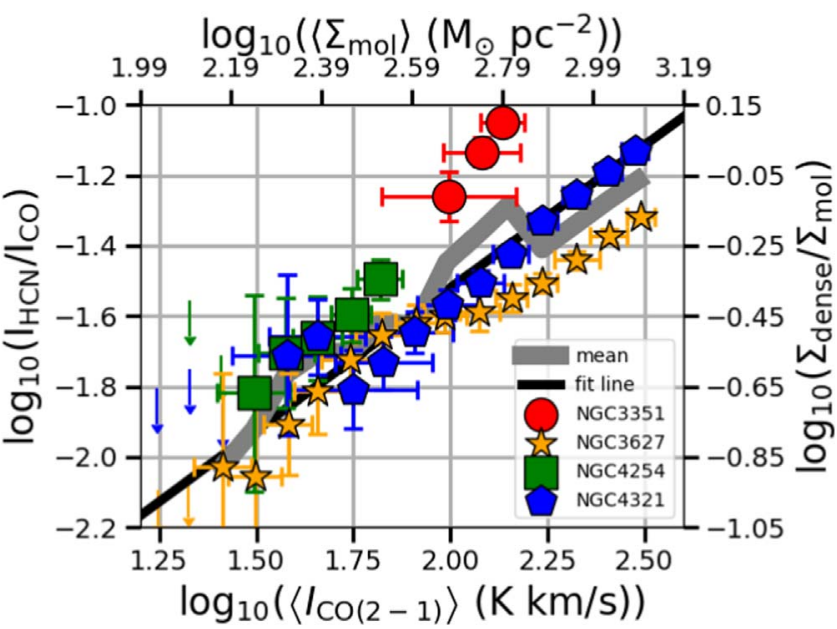

Figure 2. HCN $(1-0) / \mathrm{CO}(1-0)$, a spectroscopic tracer of $f_{\text {dense, }}$, as a function of the molecular cloud surface density averaged within $\sim 0.6 \mathrm{kpc}$ regions across our ALMA sample. The mean molecular cloud surface density is estimated from the mean cloud-scale $\mathrm{CO}(2-1)$ intensity, $\left\langle I_{\mathrm{CO}(2-1)}\right\rangle$, inside each $\sim 0.6 \mathrm{kpc}$ region. Colored points show mean $\mathrm{HCN} / \mathrm{CO}$ in bins of fixed $\left\langle I_{\mathrm{CO}(2-1)}\right\rangle$ for individual galaxies. The error bars on these points represent the average relative error for each bin. The gray line shows the mean $\mathrm{HCN} / \mathrm{CO}$ at a given $\left\langle I_{\mathrm{CO}(2-1)}\right\rangle$ combining all galaxies and weighting each galaxy equally. The black line indicates the best fit line (via the bisector method) to the binned data for all galaxies (see Table 2). Filled symbols show bins where the integrated $\mathrm{S} / \mathrm{N}$ for $\mathrm{HCN} / \mathrm{CO} \geqslant 3 \sigma$. Downward-pointing arrows show upper limits.

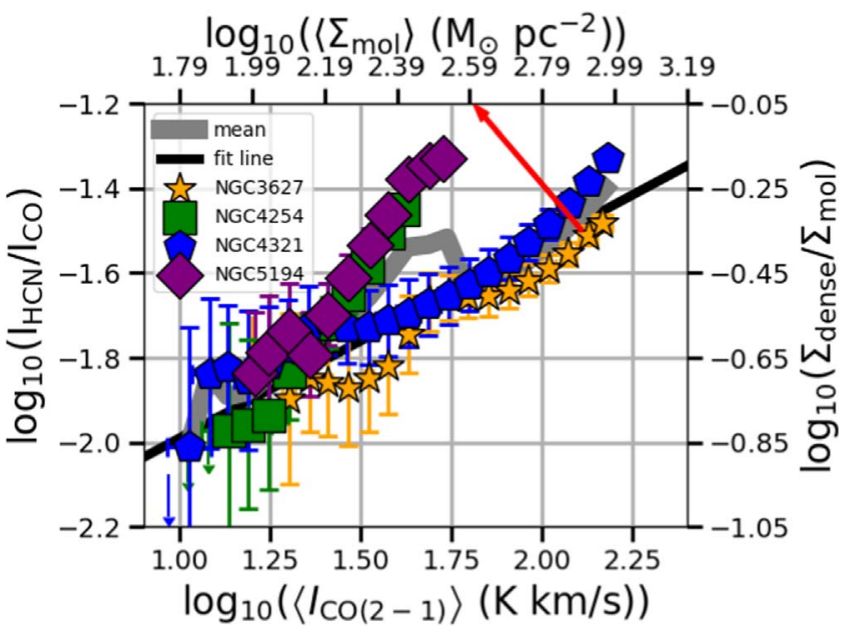

Figure 3. As for Figure 2, but now showing results averaged over $\sim 2.8 \mathrm{kpc}$ regions in the EMPIRE IRAM $30 \mathrm{~m}$ targets. The red arrow shows the effect of lowering $\alpha_{\mathrm{CO}}$ by a factor of 2, while leaving $\alpha_{\mathrm{HCN}}$ constant. Based on Sandstrom et al. (2013), such an adjustment should be appropriate for the inner part of NGC 3627 and NGC 4321 but not NGC 4254 or NGC 5194.

\subsection{Additional Checks}

To check our results, we also analyzed the ALMA HCN data at the EMPIRE common physical resolution of $2770 \mathrm{pc}$. At a fixed $\left\langle I_{\mathrm{CO}(2-1)}\right\rangle$, the ALMA and EMPIRE data differ by a mean of $15 \%$ in $\mathrm{HCN} / \mathrm{CO}$. Mostly, this offset reflects the fact that the two data sets cover different area (see Figure 1). When we match the areal coverage (i.e., consider EMPIRE only over the ALMA area), a smaller $\sim 5 \%$ difference remains.

Our CO (1-0) data (used in the denominator of $\mathrm{HCN} / \mathrm{CO}$ ) come from different sources for EMPIRE and the ALMA sample. We estimate the uncertainty associated with our choice of CO map by considering NGC 4321, for which we have ALMA, BIMA, and IRAM $30 \mathrm{~m} \mathrm{CO}(1-0)$ maps. We only
Table 2

Results Comparing $\mathrm{HCN} / \mathrm{CO}$ to $\left\langle I_{\mathrm{CO}(2-1)}\right\rangle$

\begin{tabular}{lccc}
\hline \hline HCN Data & Resolution & Rank. Corr. & Fit Slope, Intercept \\
\hline ALMA & $650 \mathrm{pc}$ & 0.83 & $0.81( \pm 0.09),-1.93( \pm 0.04)$ \\
ALMA & $2770 \mathrm{pc}$ & 0.77 & $0.41( \pm 0.04),-1.73( \pm 0.02)$ \\
EMPIRE & $2770 \mathrm{pc}$ & 0.78 & $0.55( \pm 0.05),-1.73( \pm 0.01)$ \\
\hline
\end{tabular}

Note. Relation between mean $120 \mathrm{pc} \mathrm{CO}(2-1)$ intensity, $\left\langle I_{\mathrm{CO}(2-1)}\right\rangle$, tracing cloud-scale mean surface density, and $\mathrm{HCN} / \mathrm{CO}$, tracing $f_{\text {dense. }} \mathrm{HCN}$ Data: the source of the HCN data. Rank Corr.: Spearman's rank correlation coefficient relating $\mathrm{HCN} / \mathrm{CO}$ to $\left\langle I_{\mathrm{CO}(2-1)}\right\rangle$ across all bins for all galaxies. All have $p$ values $<0.01$. Fit: linear fit to the logarithmic data (i.e., power-law fit), normalized at the lower end of the $\log _{10}\left\langle I_{\mathrm{CO}(2-1)}\right\rangle$ range for each sample. Shown in the table are the slope $(m)$ and intercept $(b)$ with associated uncertainties for the following equation: $\log _{10} \frac{\mathrm{HCN}}{\mathrm{CO}}=m \times \log _{10}\left\langle I_{\mathrm{CO}(2-1)} /\left(30 \mathrm{~K} \mathrm{~km} \mathrm{~s}^{-1}\right)\right\rangle b$. To convert into an approximate relation between $f_{\text {dense }}$ and surface density, use $f_{\text {dense }} \approx 2.3 \mathrm{HCN} / \mathrm{CO}$ and $\Sigma_{\text {mol }}\left[M_{\odot} \mathrm{pc}^{-2}\right] \approx 6.2\left\langle I_{\mathrm{CO}(2-1)}\right\rangle$.

have CARMA data for NGC 4254 so we cannot explore how CARMA compares to our other CO (1-0) sources. We repeat our complete analysis with each NGC $4321 \mathrm{CO}(1-0)$ map. At $650 \mathrm{pc}$ resolution, the $\mathrm{HCN} / \mathrm{CO}$ ratios measured using the ALMA CO map are $\approx 0.1$ dex lower than those measured using the BIMA map. At $2770 \mathrm{pc}$ resolution, the ALMA map yields $\mathrm{HCN} / \mathrm{CO}$ ratios $\approx 0.2 \mathrm{dex}$ lower than BIMA, while the EMPIRE maps yields ratios $\sim 0.25$ dex lower than BIMA. The ALMA and EMPIRE results agree within 0.06 dex. Overall, we have good confidence in the EMPIRE and ALMA measurements, but the two BIMA-based $\mathrm{CO}$ maps may have results uncertain by $\sim 0.1 \mathrm{dex}$ at $650 \mathrm{pc}$ resolution. While there are offsets between the ratio values calculated using different input data, these offsets do not change the nature of the observed trends.

\section{Results \\ 3.1. Observed Correlation between HCN/CO and Cloud-scale Surface Density}

Figures 2 and 3 show binned $\mathrm{HCN}(1-0) / \mathrm{CO}(1-0)$ as a function of $\left\langle I_{\mathrm{CO}(2-1)}\right\rangle$ for our two subsamples. $\mathrm{HCN}(1-0) /$ $\mathrm{CO}(1-0)$ and $\left\langle I_{\mathrm{CO}(2-1)}\right\rangle$ are strongly positively correlated for all galaxies in both samples. Spearman's rank correlation coefficient, $\rho$, is high for both individual galaxies $(\rho=$ $0.97-1.0)$ and the entire sample $(\rho=0.77-0.83$; Table 2$)$. The corresponding low $p$ values indicate that this correlation is unlikely to be produced by random noise. Our measurements offer strong evidence for a significant underlying relationship between our $f_{\text {dense }}$ (traced by $\mathrm{HCN} / \mathrm{CO}$ ) and cloud-scale surface density (traced by $\left.\left\langle I_{\mathrm{CO}(2-1)}\right\rangle\right)$.

Table 2 reports power-law fits (fit via the bisector method) that offer a reasonable description of each sample. We plot these as black lines in Figures 2 and 3.

These fits offer a good first-order description of the observed trends. However, we do observe substantial galaxy-to-galaxy variations in $\mathrm{HCN} / \mathrm{CO}$ at fixed $\left\langle I_{\mathrm{CO}(2-1)}\right\rangle$. The standard deviation in $\mathrm{HCN} / \mathrm{CO}$ at fixed $\left\langle I_{\mathrm{CO}(2-1)}\right\rangle$ is $0.11 \mathrm{dex}$ for the ALMA sample and 0.08 dex for the EMPIRE sample. Because of our binning approach, this reflects only the galaxy-to-galaxy scatter. If we were in a position to measure the cloud-to-cloud or region-to-region scatter within each $\left\langle I_{\mathrm{CO}(2-1)}\right\rangle$, we would expect to find more variation. 
NGC 3627 and NGC 4321 in the EMPIRE data (Figure 3) also exhibit different behavior at high and low $\left\langle I_{\mathrm{CO}(2-1)}\right\rangle$. For these galaxies, the slope relating $\mathrm{HCN} / \mathrm{CO}$ to $\left\langle I_{\mathrm{CO}(2-1)}\right\rangle$ steepens near $\log _{10}\left\langle I_{\mathrm{CO}(2-1)}\right\rangle \sim 1.75\left(\sim 55 \mathrm{~K} \mathrm{~km} \mathrm{~s}^{-1} \sim 350 M_{\odot}\right)$ and these galaxies show higher $\log _{10}\left\langle I_{\mathrm{CO}(2-1)}\right\rangle$ and lower HCN/ CO compared to the other two targets. Though we do not find similar curvature, the same two targets show a similar offset from NGC 4254 to high $\log _{10}\left\langle I_{\mathrm{CO}(2-1)}\right\rangle$ and lower HCN/CO in the ALMA observations (Figure 2). Below, we suggest that "starburst"-like conversion factors in the centers of these galaxies offer a likely explanation for this behavior.

\subsection{Implications}

The correlation between $\mathrm{HCN} / \mathrm{CO}$ and $\left\langle I_{\mathrm{CO}(2-1)}\right\rangle$ supports the idea that both quantities trace the density distribution of molecular gas. This suggests that the mean surface density of a molecular cloud and its dense gas content both reflect an underlying, environment-dependent gas density distribution.

Below, we show that this would be expected from simple models as long as cloud-scale mean surface density traces cloud-scale mean volume density. In fact, as discussed in (Leroy et al. 2017, see their Figure 5), surface and volume density do correlate in recent molecular cloud catalogs. Moreover, the molecular gas scale height in the Milky Way appears relatively constant over the inner $\sim 8 \mathrm{kpc}$ (Heyer \& Dame 2015). In short, current evidence appears to support the idea that at high resolution molecular gas surface density tracks molecular gas volume density to first order (see also Utomo et al. 2018).

Connection to Environment: The regions of galaxies with high gas and stellar surface densities and high interstellar gas pressure also tend to have high dense gas fractions, $f_{\text {dense }}$ (Usero et al. 2015; Bigiel et al. 2016; Gallagher et al. 2018). These also tend to be in the inner parts of galaxies, so the binned results in Figures 2 and 3 also roughly map to radius and stellar surface density, with the central regions of each galaxy mostly contributing to the top-right part of the relationship.

At the same time, many recent studies have shown an environmental dependence of the cloud-scale properties of molecular gas (e.g., Hughes et al. 2013; Colombo et al. 2014; Leroy et al. 2016; Faesi et al. 2018; Sun et al. 2018). Broadly, these results have the same sense as those for $f_{\text {dense. The }}$ internal pressure of molecular clouds appears to correlate with large-scale environmental gas pressure, radius, and the stellar mass of the host galaxy (Hughes et al. 2013; Schruba et al. 2018; Sun et al. 2018).

Our results directly connect these two lines of evidence. The correlation that we observe suggests that the properties of the bulk molecular gas (e.g., Hughes et al. 2013; Colombo et al. 2014; Leroy et al. 2016; Faesi et al. 2018; Schruba et al. 2018; Sun et al. 2018) and $f_{\text {dense }}$ (Gao \& Solomon 2004; Usero et al. 2015; Bigiel et al. 2016; Gallagher et al. 2018) reflect different aspects of the same environment-dependent density distribution. The sense of this correlation should broadly be that highpressure, high-surface-density, inner parts of galaxies have both high $f_{\text {dense }}$ and high cloud-scale mean surface density.

Does $\alpha_{\mathrm{CO}}$ Drive Galaxy-to-galaxy Variations? Sandstrom et al. (2013) found that the $\mathrm{CO}-$ to- $\mathrm{H}_{2}$ conversion factor $\left(\alpha_{\mathrm{CO}}\right)$ is often lower than the standard Galactic value in the inner regions of galaxies with dense, bar-fed centers. Specifically, they found 2-3 times lower $\alpha_{\mathrm{CO}}$ in the center of NGC 3627 and NGC 4321 compared to the disks. NGC 4254 (Sandstrom et al. 2013) and NGC 5194 Leroy et al. (2017) do not show central $\alpha_{\mathrm{CO}}$ depressions, and NGC 4254 may even show a central rise in $\alpha_{\mathrm{CO}}$. An arrow in Figure 3 indicates the effect of lowering $\alpha_{\mathrm{CO}}$ (but not $\alpha_{\mathrm{HCN}}$ ) by a factor of 2 . Adjusting the inner (high $\left.\left\langle I_{\mathrm{CO}(2-1)}\right\rangle\right)$ NGC 3627 and NGC 4321 points in this way would bring the different galaxies into better agreement. If $\alpha_{\mathrm{CO}}$ and $\alpha_{\mathrm{HCN}}$ change in the same way, then the points only move horizontally.

Though not a unique explanation, a low $\alpha_{\mathrm{CO}}$ due to bright diffuse (but not dense) molecular gas offers a feasible explanation for some of the offset among our targets. This change in $\alpha_{\mathrm{CO}}$ as a function of $\left.\left\langle I_{\mathrm{CO}(2-1)}\right\rangle\right)$ could also explain the curvature in the EMPIRE results for NGC 3627 and NGC 4321.

Indirect Evidence that Both Axes Trace Density: A number of recent studies have raised concerns about the ability of $\mathrm{HCN}$ to trace dense gas (e.g., Rathborne et al. 2015; Kauffmann et al. 2017; Leroy et al. 2017; Pety et al. 2017). The unknown abundance of the molecule, its uncertain opacity (JiménezDonaire et al. 2017), and possible excitation effects (Shimajiri et al. 2017) all are likely to affect the HCN-to-dense gas conversion factor. Similarly, $\left\langle I_{\mathrm{CO}(2-1)}\right\rangle$ at $120 \mathrm{pc}$ resolution may suffer from excitation effects (Koda et al. 2012). Variations in the line-of-sight depth could also introduce scatter into the relationship between mean volume density and mean surface density.

Despite these concerns, these two observables remain among our most practical tracers of $f_{\text {dense }}$ and mean cloud-scale surface density for observations of external galaxies. Our finding that they track each other is a powerful, though still indirect, evidence that both the $\mathrm{HCN} / \mathrm{CO}$ ratio and the cloud-scale $\mathrm{CO}$ intensity are meaningful tracers of gas density. Moreover, even if $\mathrm{HCN}$ traces lower density gas than is commonly assumed, the contrast between the HCN and $\mathrm{CO}$ lines still captures the shape of the density distribution to some extent.

\subsection{Expectations from Simple Models}

Our observed correlation would be expected if (1) $\mathrm{HCN} / \mathrm{CO}$ traces the fraction of gas above some threshold density, (2) $\left\langle I_{\mathrm{CO}(2-1)}\right\rangle$ traces the mean surface density of molecular clouds, and (3) the mean surface density of a molecular cloud traces its mean volume density. Here we illustrate this by integrating over some simple models of gas density distributions.

We calculate $f_{\text {dense }}$ as a function of mean density for three model gas volume density distributions" ${ }^{26}$ : (1) a "bottomheavy" power law with a slope of -2.5 , such that $d p / d n$ $\propto n^{-2.5}$; (2) a power law with $d p / d n \propto n^{-2}$ and a width of 2 dex; such a distribution has equal mass per logarithmic bin (i.e., a "top hat" in mass); and (3) a lognormal distribution with $1 \sigma$ width of 1.0 dex. Power-law and lognormal distributions are currently the most popular ways to represent volume and column density distributions (e.g., Padoan \& Nordlund 2002; Kainulainen et al. 2009; Lombardi et al. 2015).

Figure 4 shows the shape of each model distribution for several mean densities. To calculate the dense gas mass fraction, we take the ratio of mass above two thresholds, one at

\footnotetext{
${ }^{26}$ In our notation $p$ refers to the probability of finding a given piece of volume in the cloud to have density $n . d p / d n$ refers to the probability density distribution as a function of density. The mass of material at a density $n$ will be $m=n \times p$. In the upper two and lower-left panels of Figure 4 we plot $\log d m / d \log n$, illustrating the distribution of mass as a function of density.
} 

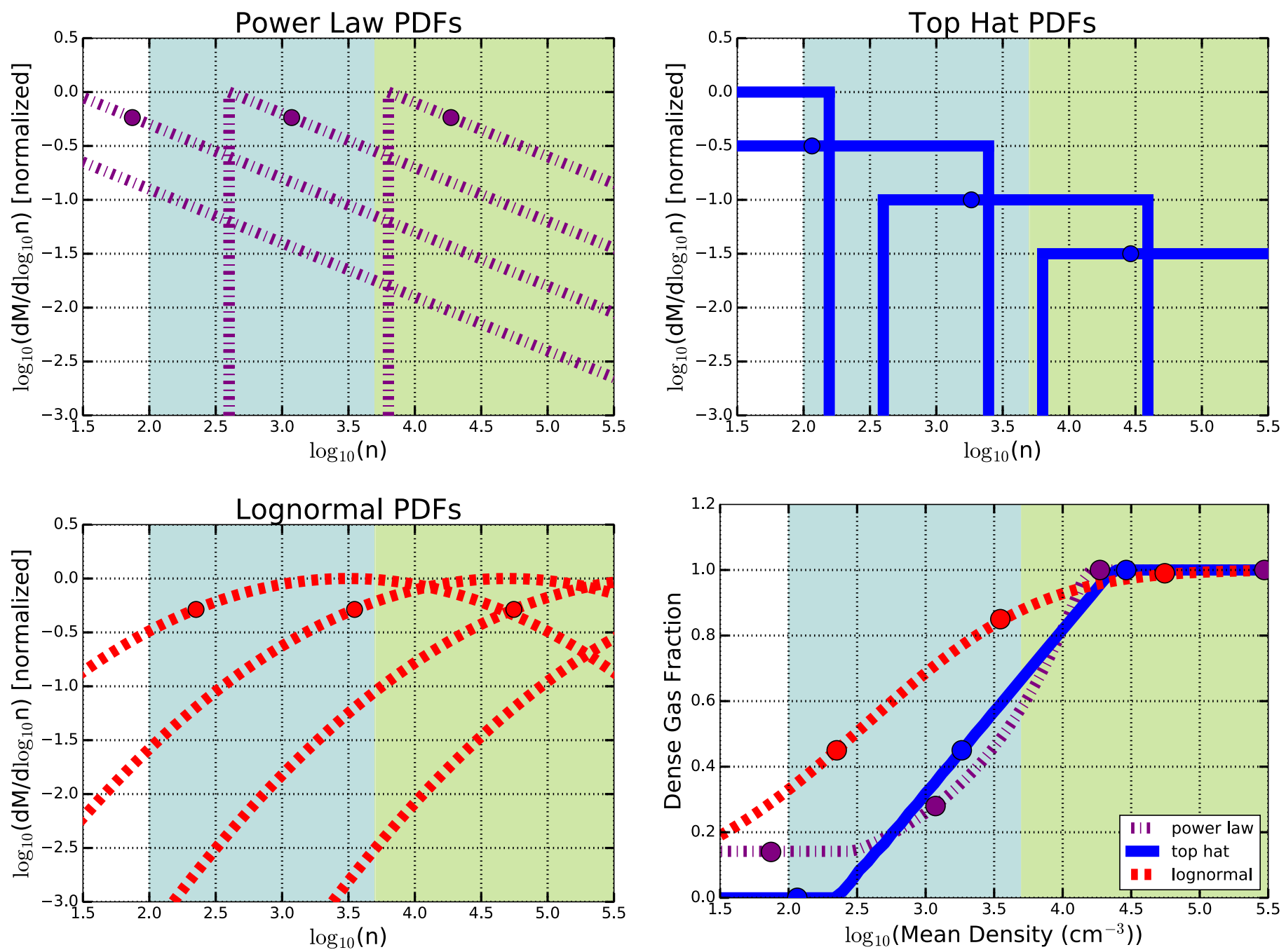

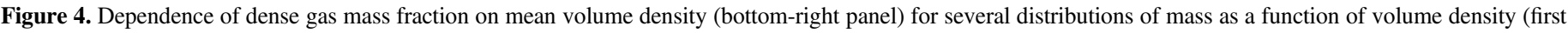

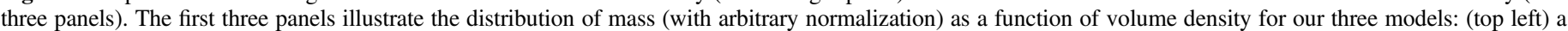

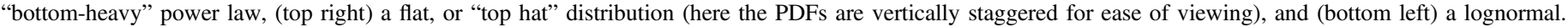

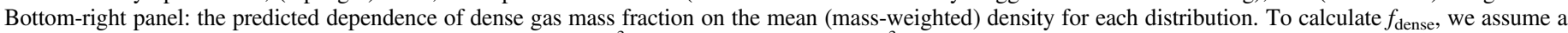

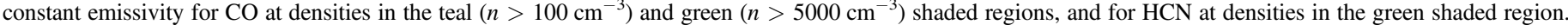

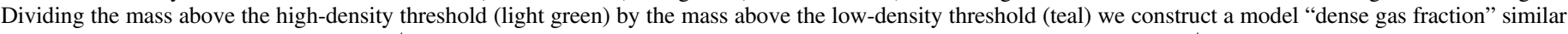

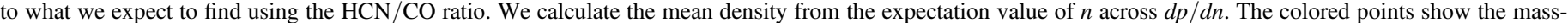
weighted mean for each illustrated density distribution.

low density (teal) and one at high density (light green). These thresholds correspond to the $n_{\text {eff }}$ of the two lines. Dividing the mass above the high-density threshold by the mass above the low-density threshold we construct a model "dense gas fraction" similar to what we expect to find using the HCN/ $\mathrm{CO}$ ratio.

To predict how $f_{\text {dense }}$ depends on mean density, we repeat the exercise for many distributions. We leave the width (when applicable) and slope of each distribution fixed, keep the CO and $\mathrm{HCN}$ density thresholds fixed, and vary only the mean density of the cloud. The lines in the bottom-right panel of Figure 4 shows the resulting $f_{\text {dense }}$ as a function of mean density (the points indicate the specific cases illustrated in the other panels).

For all distributions, Figure 4 shows that as the mean density increases, a larger and larger fraction of the gas sits above the effective density of $\mathrm{HCN}(1-0)$ (the light green region). As a result, we expect a correlation between mean density and $f_{\text {dense- }}$. At intermediate densities, where the mean density lies between the low- $(\mathrm{CO})$ and high- $(\mathrm{HCN})$ density threshold, power-lawlike scaling relations between mean density and $f_{\text {dense }}$ are common. If our observed $\left\langle I_{\mathrm{CO}(2-1)}\right\rangle$, tracing the mean cloudscale surface density, also traces the mean cloud-scale volume density, then our observations would match the expectation from these simple models.

\section{Summary}

We measure how the ratio of $\mathrm{HCN}(1-0)$ to $\mathrm{CO}(1-0)$ emission depends on the $120 \mathrm{pc}$ scale $\mathrm{CO}$ intensity, $\left\langle I_{\mathrm{CO}(2-1)}\right\rangle$, in five nearby galaxies. $\mathrm{HCN} / \mathrm{CO}$ traces the dense gas mass fraction, while $\left\langle I_{\mathrm{CO}(2-1)}\right\rangle$ measures the cloud-scale molecular gas mass surface density.

We find a strong correlation between these two quantities, albeit with differences in the shape and normalization from galaxy to galaxy. This could be expected if these two quantities trace different aspects of the same underlying distribution of 
gas densities. We illustrate this using simple model density distributions.

This result supports a view in which the large-scale structure of a galaxy shapes the local gas density distribution. Both the mean cloud-scale gas surface density, which is often measured as a property of molecular clouds, and the dense gas fraction, which is probed via spectroscopy, reflect this distribution. In this case, recent results tracing the environmental dependence of molecular cloud properties (e.g., Hughes et al. 2013; Colombo et al. 2014; Sun et al. 2018) and those showing a dependence of dense gas fraction on local disk structure (e.g., Usero et al. 2015; Bigiel et al. 2016; Gallagher et al. 2018) capture highly related aspects of the coupling between the physical state of cold gas and galactic environment.

Our analysis only scratches the surface of what can be done comparing cloud properties to density-sensitive spectroscopy. In the near future, it should be possible to expand this sample by combining PHANGS-ALMA $\mathrm{CO}$ maps with new $\mathrm{HCN}$ observations from ALMA, the Green Bank Telescope, and the IRAM $30 \mathrm{~m}$. Expanding the analysis to a suite of lines with a wide range of critical densities will better constrain the volume density distribution (Leroy et al. 2017; Gallagher et al. 2018). The CO imaging also includes information on cloud-scale dynamics, which will allow us to test how the the cloud-scale velocity dispersion and virial parameter relate to gas density and the star formation. Finally, as mentioned above, our knowledge of how HCN and similar lines trace dense gas is improving rapidly thanks to ongoing theoretical (e.g., Onus et al. 2018), extragalactic (e.g., Jiménez-Donaire et al. 2017), and Galactic studies (e.g., Kauffmann et al. 2017; Mills \& Battersby 2017; Pety et al. 2017).

We thank the anonymous referee for a fast and constructive report that improved the quality of this Letter. This Letter makes use of the following ALMA data: ADS/JAO.ALMA \#2015.1.00956.S, ADS/JAO.ALMA \#2013.1.00634.S, ADS/ JAO.ALMA \#2011.0.00004.SV. ALMA is a partnership of ESO (representing its member states), NSF (USA) and NINS (Japan), together with NRC (Canada) and NSC and ASIAA (Taiwan), and KASI (Republic of Korea), in cooperation with the Republic of Chile. The National Radio Astronomy Observatory is a facility of the National Science Foundation operated under cooperative agreement by Associated Universities, Inc. The Joint ALMA Observatory is operated by ESO, AUI/NRAO and NAOJ. M.G. acknowledges generous support from the NRAO student observing support program. The work of M.G., A.K.L., J.S., and D.U. is partially supported by the National Science Foundation under grants No. 1615105, 1615109, and 1653300. F.B. acknowledges funding from the European Unions Horizon 2020 research and innovation programme (grant agreement No. 726384). E.S. acknowledges funding from the European Research Council (ERC) under the European Unions Horizon 2020 research and innovation programme (grant agreement No. 694343). A.U. acknowledges support from Spanish MINECO grants ESP2015-68964 and AYA2016-79006. S.C.O.G. acknowledges support from the DFG via SFB 881 "The Milky Way System" (sub-projects B1, B2 and B8). M.R.K. acknowledges support from the Australian Research Council (Discovery Projects award DP160100695, and the Centre of Excellence for All Sky Astrophysics in 3 Dimensions, project CE170100013). J.M.D.K. and M.C. gratefully acknowledge funding from the German Research Foundation (DFG) in the form of an Emmy Noether Research Group (grant No. KR4801/ 1-1). J.M.D.K. gratefully acknowledges funding from the European Research Council (ERC) under the European Unions Horizon 2020 research and innovation programme via the ERC Starting Grant MUSTANG (grant agreement No. 714907). D.C. is supported by the European Union's Horizon 2020 research and innovation programme under the Marie Skłodowska-Curie grant agreement No. 702622. J.P. acknowledges support from the Program National "Physique et Chimie du Milieu Interstellaire" (PCMI) of CNRS/INSU with INC/INP, co-funded by CEA and CNES. E.R. acknowledges the support of the Natural Sciences and Engineering Research Council of Canada (NSERC), funding reference number RGPIN-2017-03987.

\section{ORCID iDs}

Molly J. Gallagher (iD https://orcid.org/0000-0001-5285-5930

Adam K. Leroy (ib https://orcid.org/0000-0002-2545-1700

Frank Bigiel (i) https://orcid.org/0000-0003-0166-9745

Diane Cormier (iD https://orcid.org/0000-0002-1046-2685

María J. Jiménez-Donaire (iD https://orcid.org/0000-00029165-8080

Annie Hughes (i) https://orcid.org/0000-0002-9181-1161 Jérôme Pety (10) https://orcid.org/0000-0003-3061-6546 Eva Schinnerer (i) https://orcid.org/0000-0002-3933-7677 Jiayi Sun (iD https://orcid.org/0000-0003-0378-4667 Antonio Usero (iD https://orcid.org/0000-0003-1242-505X Dyas Utomo (iD https://orcid.org/0000-0003-4161-2639 Alberto Bolatto (i1) https://orcid.org/0000-0002-5480-5686 Chris Faesi (i) https://orcid.org/0000-0001-5310-467X Simon C. O. Glover (iD https://orcid.org/0000-0001-6708-1317 Amanda A. Kepley (iD https://orcid.org/0000-0002-3227-4917 J. M. Diederik Kruijssen (iD https://orcid.org/0000-00028804-0212

Mark R. Krumholz (i) https://orcid.org/0000-0003-3893-854X Sharon E. Meidt (iD https://orcid.org/0000-0002-6118-4048 David S. Meier (iD https://orcid.org/0000-0001-9436-9471 Eric Murphy (iD https://orcid.org/0000-0001-7089-7325 Miguel Querejeta (iD https://orcid.org/0000-0002-0472-1011 Erik Rosolowsky (ib https://orcid.org/0000-0002-5204-2259 Toshiki Saito (ib https://orcid.org/0000-0002-2501-9328

\section{References}

André, P., Di Francesco, J., Ward-Thompson, D., et al. 2014, in Protostars and Planets VI, ed. H. Beuther et al. (Tucson, AZ: Univ. Arizona Press), 27 Bigiel, F., Leroy, A. K., Jiménez-Donaire, M. J., et al. 2016, ApJL, 822, L26 Bolatto, A. D., Wolfire, M., \& Leroy, A. K. 2013, ARA\&A, 51, 207 Carter, M., Lazareff, B., Maier, D., et al. 2012, A\&A, 538, A89 Chen, H., Gao, Y., Braine, J., \& Gu, Q. 2015, ApJ, 810, 140

Colombo, D., Hughes, A., Schinnerer, E., et al. 2014, ApJ, 784, 3

Cormier, D., Bigiel, F., Jiménez-Donaire, M. J., et al. 2018, MNRAS, 475, 3909

Evans, N. J., II, Heiderman, A., \& Vutisalchavakul, N. 2014, ApJ, 782, 114

Faesi, C. M., Lada, C. J., \& Forbrich, J. 2018, ApJ, 857, 19

Federrath, C., \& Klessen, R. S. 2013, ApJ, 763, 51

Gallagher, M. J., Leroy, A. K., Bigiel, F., et al. 2018, ApJ, 858, 90

Gao, Y., \& Solomon, P. M. 2004, ApJ, 606, 271

García-Burillo, S., Usero, A., Alonso-Herrero, A., et al. 2012, A\&A, 539, A8

Heiderman, A., Evans, N. J., II, Allen, L. E., Huard, T., \& Heyer, M. 2010, ApJ, 723, 1019

Helfer, T. T., Thornley, M. D., \& Regan, M. W. 2003, ApJS, 145, 259

Heyer, M., \& Dame, T. M. 2015, ARA\&A, 53, 583

Hughes, A., Meidt, S. E., Colombo, D., et al. 2013, ApJ, 779, 46

Jiménez-Donaire, M. J., Bigiel, F., Leroy, A. K., et al. 2017, MNRAS, 466, 49 Kainulainen, J., Beuther, H., Henning, T., \& Plume, R. 2009, A\&A, 508, L35 Kauffmann, J., Goldsmith, P. F., Melnick, G., et al. 2017, A\&A, 605, L5 
Kepley, A. A., Bittle, L., Leroy, A. K., et al. 2018, arXiv:1806.07973

Koda, J., Scoville, N., Hasegawa, T., et al. 2012, ApJ, 761, 41

Kruijssen, J. M. D., Longmore, S. N., Elmegreen, B. G., et al. 2014, MNRAS, 440, 3370

Krumholz, M. R., \& Thompson, T. A. 2007, ApJ, 669, 289

Lada, C. J., Forbrich, J., Lombardi, M., \& Alves, J. F. 2012, ApJ, 745, 190

Lada, C. J., \& Lada, E. A. 2003, ARA\&A, 41, 57

Lada, C. J., Lombardi, M., \& Alves, J. F. 2010, ApJ, 724, 687

Leroy, A. K., Hughes, A., Schruba, A., et al. 2016, ApJ, 831, 16

Leroy, A. K., Schinnerer, E., Hughes, A., et al. 2017, ApJ, 846, 71

Leroy, A. K., Usero, A., Schruba, A., et al. 2017, ApJ, 835, 217

Leroy, A. K., Walter, F., Sandstrom, K., et al. 2013, AJ, 146, 19

Lombardi, M., Alves, J., \& Lada, C. J. 2015, A\&A, 576, L1

Longmore, S. N., Bally, J., Testi, L., et al. 2013, MNRAS, 429, 987

McMullin, J. P., Waters, B., Schiebel, D., et al. 2007, in ASP Conf. Ser. 376 ,

Astronomical Data Analysis Software and Systems XVI, ed. R. A. Shaw et al. (San Francisco, CA: ASP), 127
Mills, E. A. C., \& Battersby, C. 2017, ApJ, 835, 76

Onus, A., Krumholz, M. R., \& Federrath, C. 2018, MNRAS, 479, 1702

Ossenkopf, V., \& Mac Low, M.-M. 2002, A\&A, 390, 307

Padoan, P., \& Nordlund, A. 2002, ApJ, 576, 870

Pety, J., Guzmán, V. V., Orkisz, J. H., et al. 2017, A\&A, 599, A98

Pety, J., Schinnerer, E., Leroy, A. K., et al. 2013, ApJ, 779, 43

Rahman, N., Bolatto, A. D., Wong, T., et al. 2015, ApJ, 802, 125

Rathborne, J. M., Longmore, S. N., Jackson, J. M., et al. 2015, ApJ, 802, 125

Sandstrom, K. M., Leroy, A. K., Walter, F., et al. 2013, ApJ, 777, 5

Schinnerer, E., Meidt, S. E., Pety, J., et al. 2013, ApJ, 779, 42

Schruba, A., Kruijssen, J. M. D., \& Leroy, A. K. 2018, ApJ, submitted

Schruba, A., Leroy, A. K., Walter, F., et al. 2011, AJ, 142, 37

Shimajiri, Y., André, P., Braine, J., et al. 2017, A\&A, 604, A74

Sun, J., Leroy, A. K., Schruba, A., et al. 2018, ApJ, 860, 172

Tully, R. B., Rizzi, L., Shaya, E. J., et al. 2009, AJ, 138, 323

Usero, A., Leroy, A. K., Walter, F., et al. 2015, AJ, 150, 115

Utomo, D., Sun, J., Leroy, A. K., et al. 2018, ApJL, 861, L18 